Trotz einiger Redundanzen im theoretischen Teil überzeugt der Band sowohl hinsichtlich der theoretischen Einordnung der Formen von Politikberatung als auch in der empirischen Analyse. Bezweifelt werden darf aber, ob die Autoren mit ihren eigenen Beratungsvorschlägen Erfolg haben werden: Eine verstärkte Verbindung zwischen Parlament und Bürgerkonferenzen ist in Deutschland nicht absehbar.

Helmar Schöne

\title{
Verfassungsrechtliche Strukturen direkter Demokratie: dogmatisch starke Klärung
}

\author{
Hartmann, Bernd J.: Volksgesetzgebung und Grundrechte (Münsterische Beiträge zur Rechtswis- \\ senschaft, Bd. 160), Verlag Duncker \& Humblot, Berlin 2005, 248 Seiten, € 69, 80.
}

Angeregt zu seiner Untersuchung hat Bernd J. Hartmann ein Beschluss des Bundesverfassungsgerichts von 1997, der die Verfassungsbeschwerde der Beauftragten des bayerischen Volksbegehrens „Das bessere Müllkonzept“, die sich gegen unfaire Abstimmungspropaganda staatlicher und kommunaler Amtsträger wehrte, als unzulässig verwarf. Aber seine Studie malt nicht ein farbiges Panorama vergleichbarer Fälle und ähnlicher Judikate, sondern Hartmann möchte die vom Bundesverfassungsgericht entwickelte Lösung überprüfen, und dafür geht er das Problem prinzipiell, fast möchte man sagen: fundamental, an, als Frage nach der Grundrechtsgeltung im Volksgesetzgebungsverfahren: Wann lebt der (gesetzgebende) Bürger seine grundrechtliche Freiheit aus, und wann ist der (bürgerliche) Gesetzgeber, da er Staatsgewalt ausübt, grundrechtsgebunden? Seine „an Grundsätzlichem interessierte und zum Grundgesetz geschriebene Abhandlung" (S. 132) abstrahiert konsequenterweise von den Landesverfassungen und der Staatspraxis in den Ländern (sowie erst recht von den Normen und der Wirklichkeit von Bürgerbegehren und Bürgerentscheid nach Kommunalverfassungsrecht), was insofern nicht ohne Pointe ist, als es Hartmann nicht nur um eine eventuell einzuführende Bundesvolksgesetzgebung geht - die zu der Zeit, als er seine Studie erarbeitete, in der rot-grünen Koalitionsvereinbarung stand -, sondern seine Auslegung des Grundgesetzes selbstverständlich Gültigkeit für die direkte Demokratie auf Landes- und Kommunalebene beansprucht.

In gewissem Widerspruch zu dem Abstraktionsvorsatz breitet der Verfasser erst noch eine umfassende Synopse der rechtlichen Grundlagen direktdemokratischer Sachentscheidungen in Ländern und Kommunen aus (S. 29 bis 42). Dann aber enteilt er auf die Höhen des Verhältnisses von Gesellschaft und Staat, von privatem und öffentlichem Recht, von subjektivem Recht und Kompetenz sowie von Grundrechtsberechtigung und Grundrechtsbindung. Für die Antwort auf seine fundamentale Frage arbeitet der Autor die Konturen des Modells eines grundgesetzlichen Volksgesetzgebungsverfahrens heraus, dessen einzelne Schritte er als grundrechtsberechtigten Freiheitsgebrauch (so zum Beispiel Entwurfsfertigung und Abstimmungskampf - letzteren im Gegensatz zu der eingangs erwähnten Entscheidung des Bundesverfassungsgerichts, vgl. S. 119) oder als grundrechtsgebundene Wahrnehmung staatlicher Kompetenz (so zum Beispiel Volksbegehren und Volksentscheid) einordnet. Das Grundgesetz enthält für dieses Unterfangen bekanntlich nur wenige Vor- 
schriften. Wie Hartmann die Staatsfundamentalnorm über die „Abstimmungen“ (Art. 20 Abs. 2 Satz 2 GG), welche die Volksgesetzgebung ermöglicht, dazu die Grundrechts- (Art. 1 Abs. 3 GG) und die Verfassungsbindung (Art. 20 Abs. 3 GG) der Gesetzgebung, die Homogenitätsvorgaben für die Landesverfassungen (Art. 28 Abs. 1 GG) und die Wahlrechtsgrundsätze (Art. 38 Abs. 1 GG), schließlich mit Blick auf den verfassungsändernden Gesetzgeber den änderungsfesten Kern des Grundgesetzes (Art. 79 Abs. 3 GG) auslegt, ist subtil, von beeindruckender methodischer Disziplin und weithin überzeugend.

Alle dogmatische Stärke schließt Schwächen nicht aus. Wenn Hartmann die Gleichwertigkeit von Volks- und Parlamentsgesetzen - ein zentrales und folgenreiches Problem - mit der peripheren Vorschrift des Art. 28 Abs. 1 Satz 4 GG begründet, derzufolge in (kleinen) Gemeinden an die Stelle einer gewählten Körperschaft die Gemeindeversammlung treten könne (S. 164), tauchen Zweifel auf, ob man diese Argumentation als normtextorientiert loben oder als Verkennung der Sedes materiae tadeln soll. Wie hätte der Verfasser wohl jene Grundsatzfrage beantwortet, wenn der Parlamentarische Rat dieses Nebenproblem ungeregelt gelassen hätte?

Die Folgerungen aus seiner analytischen Arbeit sind zudem manchmal abstrakt, zum Beispiel wenn er für den Volksentscheid eine Abstimmungspflicht deduziert im Unterschied zur Wahl(beteiligungs-)freiheit (S. 136 bis 149) und als Entscheidungsmaßstab das Gemeinwohl herausarbeitet im Gegensatz zum Wahlrecht, bei dem der Maßstab der Eigennutz sei (S. 149 ff.); Hartmann räumt selbst ein, dass die praktischen Auswirkungen dieser Vorgaben „begrenzt“ seien (S. 217). Mitunter sind seine Folgerungen allerdings auch konkret - so beim Verfahrensrecht -, ja geradezu handfest, wenn etwa die verschiedenen gerichtlichen Rechtsschutzmöglichkeiten für alle Verfahrensschritte erörtert werden und Hartmann wider eine oft inkonsistente Rechtsprechung (einschließlich wiederum jenes Beschlusses des Bundesverfassungsgerichts von 1997, vgl. S. 180 bis 183) dank seiner Vorarbeit systematisch überzeugende Lösungen weisen kann.

Vollständig genießen kann diese bei Bodo Pieroth in Münster entstandene juristische Dissertation nur, wer erhebliche Kenntnisse des Öffentlichen Rechts mitbringt. Politikwissenschaftlich beziehungsweise politisch wichtig ist, sich klarzumachen, welche Unterschiede es - bei allen Gemeinsamkeiten - verfassungsrechtlich doch auch zwischen Wahlen und Abstimmungen gibt, und die grundgesetzlichen Vorgaben zu sehen, die für die direkte Demokratie in Deutschland auf allen Ebenen bestehen.

Hartmann behandelt nicht mehr die herkömmliche verfassungspolitische Frage, ob man das Grundgesetz um Elemente direkter Demokratie ergänzen solle. Er geht davon aus, dass die Volksgesetzgebung auf Landes- und Kommunalebene existiert und auf Bundesebene kommen wird. Für die Gegenwart dort und die Zukunft hier will er Strukturen klären und die Spielräume des verfassungsändernden Bundesgesetzgebers ausmessen. Dies hat er mit bemerkenswerter dogmatischer Stärke geleistet. 